\title{
Um Modelo de Dimensionamento de Lotes para uma Fábrica de Refrigerantes
}

\author{
M.S. RANGEL ${ }^{1}$, D. FERREIRA ${ }^{2}$, Departamento de Ciências de Computação e \\ Estatística, IBILCE, UNESP, Rua Cristóvão Colombo 2265, 15054-000 São José \\ do Rio Preto, SP, Brasil.
}

\begin{abstract}
Resumo. Neste trabalho propomos e investigamos as propriedades de um modelo de programação inteira mista para determinar o dimensionamento de lotes de uma fábrica de refrigerantes. A fábrica produz refrigerantes de vários sabores e em diferentes tipos de embalagem. A linha de produção é tal que os vasilhames são colocados em uma esteira rolante e passam por diversos estágios. Inicialmente os vasilhames são lavados, em seguida são enchidos com uma determinada quantidade de xarope e água carbonada, seguem pela esteira onde são fechados, rotulados e empacotados. As máquinas precisam de um tempo de preparação toda vez que um novo sabor e/ou novo tamanho de vasilhame for utilizado. No modelo é considerado também a capacidade da unidade de produção, e a capacidade e disponibilidade dos tanques que armazenam o xarope.
\end{abstract}

\section{Introdução}

O desenvolvimento e a solução de modelos de programação matemática aplicados a situações do mundo real são grandes desafios para os pesquisadores no mundo e em particular no Brasil. Além das dificuldades próprias da natureza do problema a ser modelado, surgem dificuldades relativas ao relacionamento entre o pesquisador e a empresa estudada que vão desde o sigilo relativo aos dados da empresa até a necessidade de se comprovar a eficiência do uso de modelos matemáticos para a otimização dos processos. É necessário mostrar que a construção de modelos matemáticos dos processos de uma dada empresa constitui um excelente método para o entendimento das estruturas da mesma ([16]), pois para a construção de modelos mais realistas é necessário estudar e aprender as complexas inter-relações entre os vários setores da empresa.

Neste trabalho propomos um modelo de programação inteira mista para o planejamento da produção de uma fábrica de refrigerantes local. A fábrica produz refrigerantes de vários sabores e diferentes tipos de embalagem. A linha de produção é tal que as máquinas precisam de um ajuste sempre que um novo sabor e/ou novo tamanho de vasilhame for utilizado. A fábrica também faz entregas e o espaço

\footnotetext{
${ }^{1}$ socorro@dcce.ibilce.unesp.br; trabalho parcialmente financiado pela FAPESP (2000/09971-9)

2 degafer@bol.com.br, Bolsista FAPESP (1999/09938-2) no período 03/2000 a 02/2002.
} 
para armazenamento no depósito é restrito. Os produtos devem ser armazenados de forma a facilitar a remoção posterior de acordo com os pedidos.

O modelo proposto neste trabalho é um modelo de dimensionamento de lotes capacitado com tempo e custos de preparo que inclui restrições adicionais para controlar a disponibilidade de xarope e espaço de armazenamento dos produtos.

Modelos de dimensionamento de lotes com tempo de preparo são modelos difíceis, tanto do ponto de vista de obtenção de uma solução viável quando algoritmos heurísticos são utilizados, como do ponto de vista de resolução. No entanto, desconsiderar o tempo de preparo das máquinas (set-up) no dimensionamento dos lotes pode levar a modelos com soluções não implementáveis. Trigueiro et al. [14] apresentam uma discussão muito interessante sobre a necessidade de incorporar explicitamente o tempo de preparo em modelos de sistemas onde o tempo de ajuste das máquinas é importante no planejamento da produção. Existem poucos trabalhos na literatura que tratam especificamente do problema de planejamento da produção na indústria de bebidas. Podemos citar, por exemplo, Clark [2] e Toledo et al. [15] que fazem uma discussão sobre o tema diferente da abordagem proposta no presente trabalho. Clark [2] propõe um modelo que inclui variáveis inteiras para controlar a quantidade de xarope disponível nos tanques. A inclusão destas variáveis aumenta a dificuldade de solução do modelo. Clark propõe ainda uma heurística de busca em vizinhança baseada na relaxação linear do problema. O modelo proposto em Toledo et al. [15] trata simultaneamente do problema de dimensionamento de lotes e de sequenciamento dos produtos considerando várias linhas de produção. A validação preliminar do modelo é feita utilizando um sistema comercial na resolução de três problemas teste com dados reais de uma empresa de grande porte.

As demais seções deste artigo estão organizadas como segue. Na Seção 2 apresentamos com mais detalhes o sistema de produção da empresa estudada; a Seção 3 apresenta o modelo de programação inteira mista desenvolvido; na Seção 4 fazemos um estudo da estrutura combinatória do modelo proposto; a Seção 5 apresenta um estudo computacional preliminar desenvolvido para testar o grau de dificuldade do modelo. Para finalizar, apresentamos, na Seção 6, as considerações finais sobre o trabalho e as referências bibliográficas.

\section{A Linha de Produção}

A fábrica de refrigerantes em questão é uma empresa de médio porte situada na região de São José do Rio Preto-SP. A empresa é organizada em três setores: produção, armazenamento e transporte. Considerando a complexidade de otimizar simultaneamente as operações dos três setores, estudamos inicialmente o setor de produção, levando em consideração a existência de um espaço limitado para armazenamento dos produtos.

A empresa produz refrigerantes em 12 sabores que são embalados em diferentes tipos de vasilhames. São utilizadas garrafas de plástico descartáveis e garrafas de vidro que precisam ser esterilizadas antes de receberem o líquido. Existem no total 10 tipos diferentes de tamanhos entre as embalagens de plástico e as de vidro. Os insumos necessários para a produção são: xarope de diversos sabores, vasilhames 
descartáveis e vasilhames recicláveis, rótulos variados, tampas e água gaseificada.

A produção dos refrigerantes é realizada em três etapas: preparo do líquido, ajuste das máquinas e finalização do produto.

Os xaropes são preparados em quantidades pré determinadas (1310 litros), chamadas de "tachadas", com capacidade para produzir 7860 litros de refrigerante. O xarope utilizado na produção dos refrigerantes é armazenado em tanques que são ligados através de mangueiras especiais às linhas de produção. A fábrica possui três tanques pequenos e quatro tanques grandes. Os tanques devem trabalhar com uma quantidade mínima de líquido suficiente para cobrir a hélice que mistura os ingredientes e assim garantir a homogeneidade do xarope. Na linha de produção, um proporcionador mistura o xarope composto com água tratada. Esta mistura recebe gás carbônico e se torna a bebida que vai para a máquina que enche os vasilhames.

As máquinas são ajustadas inicialmente para produzir refrigerantes de um determinado sabor em um determinado tamanho. Se for necessário fabricar outro tipo de refrigerante é preciso parar a linha de produção e fazer ajustes nas máquinas para produzir um item de outro sabor e/ou tamanho.

Toda a produção da fábrica é realizada por três linhas de produção. Uma linha de produção é constituída por uma esteira rolante e diversas máquinas alinhadas em série. As máquinas são utilizadas para esterilizar os vasilhames, encher as garrafas com o líquido, fechar, rotular, codificar e empacotar os refrigerantes. Ao final do processo, os pacotes de refrigerantes são colocados nos paletes e estocados. Existe apenas uma entrada e uma saída de vasilhames. Um esquema do setor de produção da fábrica está ilustrado na Figura 1.

O gargalo de uma linha de produção é a máquina que enche os vasilhames (enchedora), pois é a sua capacidade que determina a produção. A máquina enchedora possui várias válvulas, o que possibilita o enchimento de mais de uma garrafa quase que simultaneamente. A enchedora das linhas de produção 1 e 2 possuem 42 válvulas e da linha 3 possui 64 válvulas. A parte pós-enchedora (fechar, rotular, codificar, empacotar) deve ter capacidade maior que a da enchedora, assim como a parte pré-enchedora (lavagem, preparo do líquido), pois se a enchedora estiver trabalhando bem o restante da linha de produção deve acompanhá-la.

Os pedidos de refrigerantes são recebidos diariamente. O gerente da produção anota os pedidos do dia anterior no início do $1^{\circ}$ turno de cada dia, verifica a parcela do pedido que pode ser atendida usando produtos em estoque e determina o que será produzido. O espaço para o armazenamento dos refrigerantes é restrito.

O problema que estamos abordando neste artigo é a determinação de quanto produzir em cada dia de forma a satisfazer a demanda e minimizar os custos de produção e armazenamento.

\section{O Modelo Matemático}

Discutimos a seguir o modelo matemático proposto para o planejamento da produção da fábrica de refrigerantes (FR) descrito na seção anterior. Este modelo retrata matematicamente o planejamento considerando apenas uma linha de produção, 


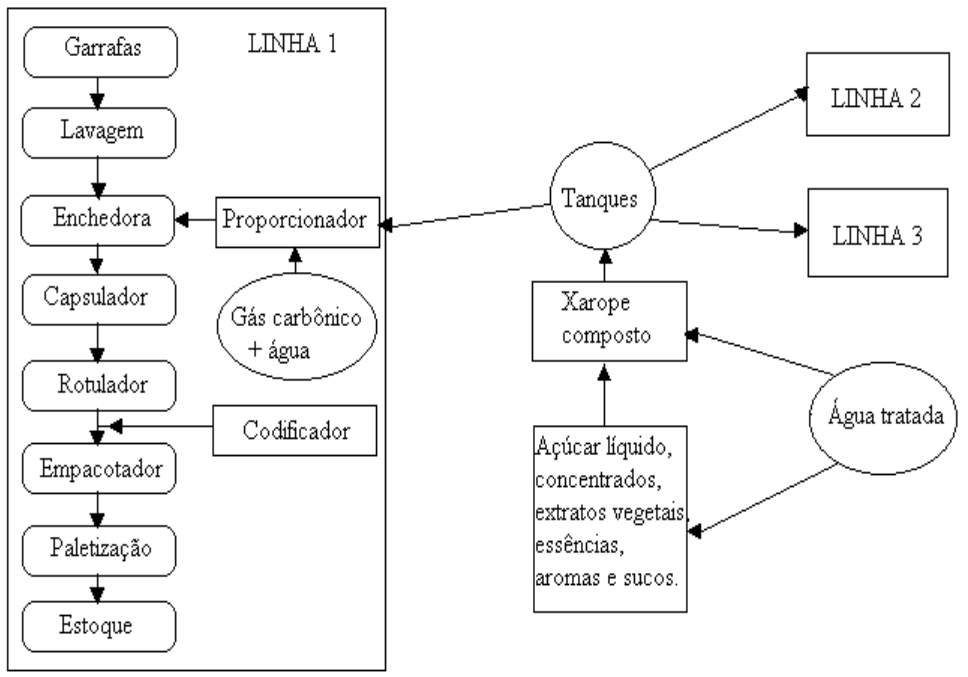

Figura 1: Esquema do setor de Produção da Fábrica de Refrigerantes

tempo e custo de preparo das máquinas. Ferreira [7] apresenta um modelo onde são incluídas também variáveis de inicialização das máquinas (start-up variables).

O planejamento consiste em determinar a quantidade de refrigerante que será produzida de forma a satisfazer a demanda do mercado, não ultrapassando as capacidades da fábrica e com objetivo de minimizar os custos de produção, armazenamento e preparo de máquinas. Considerando que a fábrica produz $s$ sabores de refrigerantes em $q$ tamanhos e em $t$ períodos, podemos modelar o problema usando os índices e variáveis definidos a seguir.

Índices:

$i$ : sabor, $i=1, \ldots, s ; j$ : tamanho, $j=1, \ldots, q ; t$ : período, $=1, \ldots, n$;

\section{Variáveis:}

$x_{i j t}=$ número de caixas de refrigerante do sabor $i$, de tamanho $j$, produzidas no período $t$

$y_{i t}=$ número de tachadas de xarope do sabor $i$ usado no período $t$;

$e_{i j t}=$ número de caixas de refrigerantes do sabor $i$, de tamanho $j$, em estoque ao final do período $t$;

$z_{i t}=\left\{\begin{array}{l}1, \text { se a máquina estiver ajustada para a produção do sabor } i \text { no período } t \\ 0, \text { caso contrário; }\end{array}\right.$

$w_{j t}=\left\{\begin{array}{l}1, \text { se a máquina estiver ajustada para a produção do tamanho } j \text { no período } t \\ 0, \text { caso contrário. }\end{array}\right.$

Dados: Considera-se conhecidos os seguintes valores:

$b_{i j}=$ número necessário de litros de xarope na produção de uma caixa de refrigerante do sabor $i$, de tamanho $j$; 
$k_{i j}=$ tempo de produção, em horas, de uma caixa de refrigerante do sabor $i$, de tamanho $j$;

$o_{i}=$ tempo de preparação, em horas: troca para o sabor $i$;

$f_{j}=$ tempo de preparação, em horas: troca para o tamanho $j$;

$h_{t}=$ tempo disponível no período $t$, em horas;

$c_{i j}^{p}=$ custo de produção de uma caixa de refrigerante do sabor $i$, de tamanho $j$;

$c_{j}^{a}=$ custo de armazenamento de uma caixa de refrigerante de tamanho $j$;

$c_{i}^{s a b}=$ custo de preparação: troca para o sabor o $i$;

$c_{j}^{\text {tam }}=$ custo de preparação: troca para o tamanho $j$;

$d_{i j t}=$ demanda em número de caixas de refrigerante do sabor $i$, de tamanho $j$, no período $t$;

$c_{t}=$ capacidade de produção do período $t$, em número de tachadas;

$q_{\min }\left(q_{\max }\right)=$ quantidade mínima (máxima) de xarope no tanque;

$l=$ capacidade do estoque em metros cúbicos;

$a_{j}=$ espaço ocupado, em metros cúbicos, por uma caixa de refrigerante de tamanho $j$ no estoque;

$M=$ constante de valor alto.

Propomos, então, um modelo de programação inteira mista que é dado por:

$$
\min \sum_{\mathrm{i}=1}^{\mathrm{s}} \sum_{\mathrm{j}=1}^{\mathrm{q}} \sum_{\mathrm{t}=1}^{\mathrm{n}}\left(\mathrm{c}_{i j}^{p} \mathrm{x}_{i j t}+\mathrm{c}_{j}^{a} \mathrm{e}_{i j t}+\mathrm{c}_{i}^{s a b} z_{i t}+\mathrm{c}_{j}^{t a m} w_{j t}\right)
$$

Sujeito a:

$$
\begin{gathered}
\sum_{\mathrm{j}=1}^{\mathrm{q}} b_{i j} x_{i j t}=1310 y_{i t}, \quad i=1, \ldots, s ; t=1, \ldots, n . \\
\sum_{\mathrm{i}=1}^{\mathrm{S}} y_{i t} \leq c_{t}, \quad t=1, \ldots, n . \\
\sum_{\mathrm{i}=1}^{\mathrm{S}} \sum_{\mathrm{j}=1}^{\mathrm{q}} k_{i j} x_{i j t}+\sum_{\mathrm{i}=1}^{\mathrm{S}} o_{i} z_{i t}+\sum_{j=1}^{\mathrm{q}} f_{j} w_{j t} \leq h_{t}, t=1, \ldots, n . \\
x_{i j t} \leq M z_{i t}, \quad \mathrm{i}=1, \ldots, s ; j=1, \ldots, q ; t=1, \ldots, n . \\
x_{i j t} \leq M w_{j t}, \quad \mathrm{i}=1, \ldots, s ; j=1, \ldots, q ; t=1, \ldots, n . \\
+x_{i j t}=d_{i j t}+e_{i j t}, \quad i=1, \ldots, s ; j=1, \ldots, q ; t=1, \ldots, n . \\
\sum_{\mathrm{i}=1}^{\mathrm{s}} \sum_{\mathrm{j}=1}^{\mathrm{q}} a_{j} e_{i j t} \leq l, \quad t=1, \ldots, n . \\
q_{\text {min }} z_{i j} \leq y_{i t} \leq q_{\max } z_{i j}, \quad i=1, \ldots, s ; t=1, \ldots, n . \\
x_{i j t} \geq 0, y_{i t} \geq 0, e_{i j t} \geq 0, z_{i t}=0 \text { ou } 1, w_{j t}=0 \text { ou } 1, \\
i=1, \ldots, s ; j=1, \ldots, q ; t=1, \ldots, n .
\end{gathered}
$$


O objetivo é definir um programa para a produção dos refrigerantes que minimize os custos de produção, os custos de armazenamento, e os custos de preparo das máquinas. As restrições de capacidade (3.1), (3.2) e (3.3) modelam, respectivamente, que a quantidade de xarope utilizada na produção dos refrigerantes deve ser igual à quantidade de xarope disponível no período, isto é todo o xarope preparado deve ser utilizado na produção; que a quantidade total de tachadas utilizadas na produção dos refrigerantes não deve ultrapassar a capacidade da fábrica, medida em número de tachadas; e que o tempo de produção dos refrigerantes, mais o tempo que requer o preparo das máquinas (troca de sabor e/ou tamanho) tem que ser menor ou igual ao tempo disponível para a produção em cada período. As restrições de preparo (3.4) e (3.5) garantem que a produção de um refrigerante do sabor $i$, de tamanho $j$, no período $t$ seja feita apenas se as máquinas estiverem ajustadas para tal. As restrições (3.6), (3.7) e (3.8) garantem, respectivamente, o balanceamento entre a produção e o estoque, que o espaço limitado para armazenamento dos refrigerantes seja respeitado e a quantidade mínima (e máxima) de xarope presente nos tanques caso haja produção do sabor $i$ no período $t$.

As variáveis $z_{i t}$ e $w_{j t}$ são conhecidas na literatura como variáveis de preparação das máquinas (set-up variables [14]). Neste modelo consideramos que o tempo de ajuste da máquina para um determinado sabor (tamanho) é independente do sabor (tamanho) usado anteriormente, de acordo com a prática realizada na empresa estudada.

Considerando apenas os conjuntos de restrições (3.3), (3.4) e (3.6) obtemos um modelo que é conhecido na literatura como modelo de planejamento da produção multi-item capacitado com tempos de preparo (PMC) (Multi-item Production Planning with set-up [14]).

Observe que o modelo (FR) permite a produção de refrigerantes de sabores e tamanhos diferentes em um mesmo período. Uma importante questão que surge é como fazer o sequenciamento de itens diferentes que são produzidos em um mesmo período. No presente trabalho, estamos supondo que o planejamento será feito em dois estágios. Um estágio determina o dimensionamento dos lotes (estágio A), e outro estágio (estágio B), feito antes ou depois do estágio A, determina a sequência em que os itens serão produzidos. O modelo (FR) é usado no estágio A. Uma revisão de métodos de solução para o problema de sequenciamento de itens, estágio B, é dada em [12]. Modelos de planejamento da produção que incluem o sequenciamento e o dimensionamento dos lotes simultaneamente podem ser vistos em [5] e [15].

\section{Estrutura Combinatória do Modelo (FR)}

O uso de planos de corte na solução de problemas de programação inteira e inteira mista tem se mostrado bastante eficiente para uma ampla classe de problemas. Além de métodos gerais para a geração de inequações válidas, como, por exemplo, o Método de Gomory ([10] e [11]), uma importante ferramenta que tem sido utilizada na identificação de inequações válidas é explorar a estrutura geométrica ou combinatória do problema a ser resolvido para gerar facetas ou faces de dimensões altas. Exemplos de problemas que tiveram sua estrutura poliédrica definida por tal método 
são: problemas de fluxo em redes com custo fixo, problema da mochila e problemas de planejamento da produção. Estas inequações podem ser úteis na solução de problemas mais gerais onde problemas específicos são identificados como subestruturas na formulação do mesmo (ver [9], [13] e referências citadas). A eficiência do uso de facetas e faces para o problema de fluxo em redes com custo fixo (Flow cuts) e para o problema da mochila 0 ou 1 (Cover cuts) como planos de corte na solução de problemas de programação inteira mista 0 ou 1 tem sido demonstrada por diversos trabalhos na literatura (ver, por exemplo, [6] e [9]).

A geração automática de planos de corte está incluída em sistemas de otimização comerciais mais recentes, como por exemplo no CPLEX [8] e em sistemas de pesquisa como o bc-opt [4]. O efeito das rotinas de planos de corte incluídas nestes sistemas vai depender da estrutura combinatória do problema que está sendo resolvido, e da capacidade do sistema em identificar tal estrutura. Muitas vezes, a identificação de uma subestrutura em um problema mais geral envolve a reformulação automática de um subconjunto das equações ou inequações que definem a região viável do problema.

Analisando a estrutura combinatória do modelo (FR) é possível identificar subestruturas que podem ser úteis na geração de inequações válidas para o modelo. Se tomarmos as restrições de preparação (3.4), juntamente com as restrições de balanço (3.6), e fixarmos um valor de $i$ e $j$ podemos ver que elas definem a região factível do problema de planejamento da produção de um único item não capacitado com variáveis de preparação (PUNC). Isto é, o modelo (FR) contém uma cópia de (PUNC) para cada produto (cada combinação de sabor/tamanho). Estes produtos são interligados pelas restrições de capacidade (3.3).

A estrutura poliédrica do problema (PUNC) está muito bem estudada na literatura. De fato, o envoltório convexo do problema é conhecido, e as inequações que o definem podem ser utilizadas como planos de corte para eliminar a solução da relaxação linear em um algoritmo do tipo branch and cut [11]. Uma discussão mais ampla sobre inequações válidas para problemas de planejamento da produção pode ser vista em [3] e referências citadas.

\section{Problemas Teste e Resultados Computacionais}

O modelo (FR) apresentado na Seção 3 foi definido considerando a produção de refrigerantes de s sabores, em q tamanhos e em n períodos, envolvendo $n(2 s q+2 s+q)$ variáveis das quais $[n s(2 q+1)]$ são variáveis não negativas e $[n(s+q)]$ são binárias. O total de restrições é $[\mathrm{n}(3 \mathrm{~s}(\mathrm{q}+1)+3)]$. Estes parâmetros podem gerar problemas de grande escala dependendo do número de sabores, tamanhos e períodos considerados. Por razões administrativas, a empresa estudada não pode disponibilizar dados reais para que pudéssemos testar a validade do modelo proposto. Em vista disto, construímos um algoritmo para gerar aleatoriamente dados de problemas que permitissem um estudo da dificuldade computacional da resolução do modelo (FR).

A obtenção de problemas teste viáveis para este modelo foi uma tarefa complicada. Em problemas de dimensionamento de lotes onde o tempo de preparo não é considerado nas restrições de capacidade, a viabilidade do mesmo pode ser verifi- 
cada comparando a demanda acumulada com a capacidade acumulada. Quando o tempo de preparo é considerado explicitamente no modelo, a questão de verificar a viabilidade de um problema é classificado como de complexidade NP-difícil ([14]). Os parâmetros utilizados na geração de dados foram estabelecidos através de testes computacionais e baseados nos parâmetros usados em [1]. Para definir a demanda, estabelecemos que se um problema teste envolvesse mais que quatro períodos, a demanda dos primeiros quatro períodos seria gerada no intervalo [0, 100] e a dos demais períodos no intervalo [500, 700]. Uma descrição detalhada do processo e dos parâmetros usados na geração aleatória dos dados está descrita em [7].

Para avaliar o grau de dificuldade do modelo (FR) proposto, fizemos um estudo computacional preliminar usando os parâmetros padrões do sistema de otimização CPLEX 7.1 [8] para Windows, em um PC Pentium III de 1 Ghz e $256 \mathrm{Mb}$ de memória RAM. O CPLEX é um sistema de otimização geral, que pode ser usado para resolver problemas de programação linear, quadrático e inteiro misto. O módulo de solução de problemas de programação inteira misto é bastante sofisticado e o algoritmo branch and cut incluído contém rotinas de pré-processamento, heurísticas, e geração de diversos tipos de planos de corte, entre eles Cortes de Gomory, Cortes de cobertura (Cover Cuts), Cortes de fluxo (Flow cuts), e Cortes de Caminho de Fluxo (Flow Path Cuts). E importante observar que as inequações que definem os cortes de caminho de fluxo fazem parte da definição do envoltório convexo do problema PUNC descrito na Seção 4. Uma discussão mais ampla sobre o algoritmo branch and cut pode ser encontrada em [4], [9], [11] e [13].

Utilizamos no experimento dois conjuntos, de nove problemas teste cada, considerando duas dimensões diferentes. O primeiro, DIM1, com 48 itens $(s=12$ e $q=4)$ e horizonte de planejamento de 15 dias e o segundo, DIM2, com 96 itens $(s=12$ e $q=8)$ e horizonte de planejamento de 36 dias. Os resultados obtidos estão descritos na Tabela 1 abaixo. Apenas três classes de planos de corte foram gerados: Cortes de Gomory, Cortes de fluxo, e Cortes de Caminho de Fluxo, sendo que para alguns problemas teste não foram geradas as inequações de Caminho de Fluxo.

Observamos que os problemas do conjunto DIM1 são facilmente resolvidos, com um tempo médio de CPU de 28,98 segundos, examinando em média 207,44 nós, e considerando um GAP de integralidade 0,01\%. Foram utilizados em média 717 planos de corte no processo de solução representando $26,12 \%$ do número de restrições do problema.

Os problemas do conjunto DIM2 são difíceis de serem resolvidos. A solução ótima não foi obtida considerando um tempo máximo de $30 \mathrm{~min}$ de CPU. Dentro deste limite de tempo foram examinados uma média de 3644,71 nós, com um GAP de integralidade médio de $0,053 \%$. Foram utilizados em média 1759,78 planos de corte no processo de solução representando 14,95\% do número de restrições do problema.

Repetimos o experimento para dois problemas do conjunto Dim2 (P28 e P30), estendendo o tempo de resolução para aproximadamente 3 horas e 2,5 horas respectivamente. Dentro deste limite de tempo o GAP anterior foi reduzido em apenas 0,01\% para os dois problemas, com uma pequena variação no número de planos de 
corte gerados. Isto indica a necessidade de se buscar métodos mais eficientes para a solução deste modelo.

\begin{tabular}{|l|l|l|l|l|}
\hline \multicolumn{5}{|c|}{ Tabela 1: Resultados Médios } \\
\hline Problema & CPU & No de Nós & GAP \% & Cuts \% \\
\hline Dim1 & 28,98 s & 207,4 & 0,001 & 26,12 \\
\hline Dim2* & 30min & 3644,71 & 0,053 & 14,95 \\
\hline
\end{tabular}

\title{
6. Considerações finais
}

Neste trabalho apresentamos um modelo de programação inteira mista para a determinação dos lotes de produção de uma fábrica de refrigerantes. Fizemos um estudo da estrutura combinatória do modelo proposto e apresentamos um estudo computacional preliminar usando o software CPLEX 7.1 para avaliar o grau de dificuldade do modelo. Os resultados obtidos, apesar de limitados, indicam a necessidade de se desenvolver técnicas específicas para a solução deste modelo. Propor algoritmos heurísticos que explorem a estrutura combinatória do modelo, bem como identificar outras classes de planos de corte são tópicos que podem melhorar o processo de solução do modelo. Uma revisão do modelo para incorporar o sequenciamento da produção e as três linhas de produção da fábrica é uma outra importante questão que deve ser abordada em trabalhos futuros.

\section{Agradecimentos}

Gostaríamos de agradecer ao Prof. Dr. Trajano Pires da Nóbrega Neto (UnespSJRP) por nos apresentar o problema, ao Prof. Dr. Marcos Arenales (USP-São Carlos) por sugestões iniciais na construção do modelo para o problema (FR), e aos revisores pelos comentários e sugestões feitos à versão inicial deste artigo.

\begin{abstract}
This paper investigates the properties of a mixed integer model applied to solve the production-planning problem of a soft drink company. The company produces soft drink bottles in different flavors and sizes. A production unit is made up of a conveyor belt and machines that wash the bottles, fill them with a combination of syrup and carbonic gas, and then seal, label and pack them. The production of a different flavor and/or size involves machine set-up time and costs. It is also taken in account the capacity of the production unit, and the capacity and availability of syrup mixing tanks.
\end{abstract}

\section{Referências}

[1] S.A. Araújo e M.N. Arenales, Problema de dimensionamento de lotes monoestágio com restrição de capacidade: modelagem, método de resolução e resultados computacionais, Pesquisa Operacional - SOBRAPO, 20, No. 2 (2000), 287-306.

[2] A.R. Clark, "Diminishing-neighbourhood search in canning-line production planning", Relatório Técnico, Faculty of Computing, Engineering and Mathematical Sciences, University of The West of England, Bristol, UK, 2001. 
[3] M. Constantino, A polyhedral approach to a production planning problem, Annals of Operations Research, 96 (2000), 75-95.

[4] C. Cordier, H. Marchand, R. Laundry and L. Wolsey, bc-opt: A branch and cut code for mixed integer programs, Mathematical Programming, 86 (1999), 335-354.

[5] A. Drexl and A. Kimms, Lot sizing and scheduling - survey and extensions, European Journal of Operational Research, 99 (1997), 221-235.

[6] K. Darby-Dowman and S. Rangel, The application of preprocessing and cutting planes for a class of production planning problems, Computational Optimization and Applications, 11 (1998), 297-318.

[7] D. Ferreira, "Um Modelo de Dimensionamento de Lotes Aplicado à Indústria de Bebidas", Dissertação de Mestrado, PPG Matemática Aplicada, IBILCE, UNESP, S.J. do Rio Preto, SP, 2002.

[8] Ilog - "Using the CPLEX Callable Library", ILOG, 2001

[9] K.L. Hoffman, Combinatorial optimization: current successes and directions for the future, Journal of Computational and Applied Mathematics, 124 (2000), 341-360.

[10] H. Marchand et al., Cutting planes in integer and mixed integer programming, Discrete Applied Mathematics, 123 (2002), 397-446.

[11] G.L. Nemhauser and L.A. Wolsey, "Integer and Combinatorial Optimization", John Wiley Sons, 1988.

[12] M. Pinedo, "Scheduling: Theory, Algorithms and Systems", Prentice Hall, 1995.

[13] S. Rangel, "Solving Integer Programming Problems using Preprocessing and Cutting Planes: Theory and Implementation of Branch and Cut", Tese de doutorado, Brunel University, Uxbridge, Inglaterra, 1995.

[14] W.W. Trigeiro, L.J. Thomas e J.O. Mcclain, Capacitated lot sizing with setup times, Management Science, 35, No. 3 (1989), 353-366.

[15] C.F.M. Toledo, P. França e R. Morabito, Proposta de um modelo conjunto de programação da produção e dimensionamento de lotes aplicado a uma indústria de bebidas, em "Anais do XXII ENEGEP", Curitiba, PR, outubro, 2002.

[16] H.P. Williams, "Model Building in Mathematical Programming", John Wiley \& Sons, 1991. 